\title{
Experimental investigation of residual saturation in mixed-wet porous media using a pore-scale approach
}

\author{
Ruthut Larpudomlert • Victor A. Torrealba • \\ Zuleima T. Karpyn · Phillip Halleck
}

Received: 7 September 2012/ Accepted: 28 August 2013/Published online: 28 September 2013

(C) The Author(s) 2013. This article is published with open access at Springerlink.com

\begin{abstract}
Wettability is an important factor in terms of flow distribution and the amount of oil left behind in a petroleum reservoir after primary, secondary, and tertiary recovery processes. In fact, most alkaline flooding operations are aimed at wettability reversal. Therefore, a good understanding of how wettability, and other relevant factors (e.g., fluid saturation and porosity), affects displacement efficiency and fluid distribution at the pore-scale can lead to successful predictions of flow properties at the macroscale. In this study, pore-scale two-phase fluid flow in a synthetic mixed-wet granular media was investigated using X-ray microCT. Analysis of residual fluid structures in granular media composed of a mixture of glass and plastic beads (ranging from 0.4 to $0.6 \mathrm{~mm}$ in diameter) showed that the number of trapped water blobs was 2.4 times greater than that of oil, whereas most of the blobs were between 0.0001 and $0.001 \mathrm{~mm}^{3}$ in size (i.e., 100 times smaller than in the case of uniform wettability), and were smaller than the mean pore volume $\left(0.03 \mathrm{~mm}^{3}\right)$. The ratio of surface area to blob volume showed a slight tendency of water blobs to wet a higher surface area of the grains than oil blobs, for blob volumes larger than the mean pore size; this phenomenon can be attributed to stronger wetting affinity of the glass grains to the water phase than that of the plastic grains to the oil phase. Furthermore, statistical analysis of the distance between residual oil and water blobs to each solid surface confirms preferential
\end{abstract}

R. Larpudomlert · V. A. Torrealba .

Z. T. Karpyn $(\bowtie) \cdot$ P. Halleck

John and Willie Leone Family Department of Energy and

Mineral Engineering, EMS Energy Institute, The Pennsylvania

State University, University Park, PA 16802-5000, USA

e-mail: ZKarpyn@psu.edu wetting affinity of oil and water to plastic and glass surfaces, respectively.

Keywords Wettability - Fluid trapping - Micro computed tomography $\cdot$ Multiphase $\cdot$ Pore-scale

\section{Introduction}

The study of fluid transport in subsurface is important in earth sciences applications, like geochemistry, ground water remediation, and petroleum engineering. In the area of hydrocarbon recovery, the industry has turned to enhanced oil recovery techniques in which complex chemical techniques are used to alter the original fluid and/ or rock properties to increase the production of the remaining oil and gas in hydrocarbon reservoirs. However, these processes depend on pore-level heterogeneities and the ability of the injectant to contact the rock and in situ fluids. Thus, it is essential to understand factors such as interfacial tension, capillary forces, and wettability to correctly predict fluid mobility and recovery efficiency Speight (2009).

Over the last several decades, various research studies have been conducted with the purpose of quantifying and validating flow properties and transport mechanisms. However, given the difficulty of obtaining direct pore-scale measurements, pore-level models have been used to predict both flow properties and transport mechanisms. These include: network models, designed as simplified representations of a pore system consisting of pore bodies and pore throats; Lattice-Boltzmann models (LB), used to calculate single-phase and two-phase flow patterns van Dijke and Piri (2007); and mesh-free Lagrangian particle methods, such as smooth particle hydrodynamics numerical models 
(SPH), developed to simulate multicomponent immiscible and miscible flow in porous media Tartakovsky and Meakin (2006).

Network modeling has been successfully used in various studies. Suicmez et al. (2008) studied the effects of wettability for water-wet, weakly water-wet, oil-wet, and weakly oil-wet media and pore-level displacement on hydrocarbon trapping, using three-phase-flow pore-scale network modeling. They found that as the medium becomes more oil-wet, the amount of oil trapped by water in the presence of gas increases. Zhao et al. (2010) also used network modeling to study the effect of wettability on oil recovery after water flooding, focusing on the effects of initial water saturation, contact angle distribution and oilwet fraction on oil recovery. Similarly, Jackson et al. (2003) studied the effect of wettability on water flooding at the reservoir scale using a network model in conjunction with reservoir scale conventional simulations. The threedimensional network model was derived from Oren et al. (1997) to represent a sample of Berea sandstone in a study suggesting that the assumption of uniform wettability leads to an underestimation of oil recovery. Oren et al. (1997) constructed a 3-D sandstone model as an input for a twophase model to simulate primary drainage and water injection for water-wet and mixed-wet systems with the purpose of predicting relative permeability and electrical conductivity. Yet, there is still concern with regard to extending a simplified network model technique to the field scale due to the difficulty of adequately describing the complex heterogeneous nature of porous systems.

Recently, X-ray microCT (MCT) has made it possible to obtain high-resolution three-dimensional maps of porous structures, and thus provides better insight into the porescale processes taking place during fluid flow experiments from direct three-dimensional imaging and resource data, which allows the development of better network models. MCT is a non-invasive technique that reveals the internal structure of an object by capturing variations in density and atomic composition. Thus, MCT images give a representation of the pore geometry of a system Chaouki et al. (1997) and Karpyn et al. (2010). In dry granular samples, the strong contrast between the solid phase and the pore space provides information for studying the porosity distribution of the system Ketcham and Carlson (2001).

Additionally, MCT images have been used to construct accurate three-dimensional networks for predicting the transport behavior and characteristics of porous media. Jiang et al. (2007) used network-extraction techniques based on MCT images to extract a geometrical and topological network representing the pore structures of three sandstone core samples. The set of algorithms developed by Jiang was designed to improve the efficiency of common thinning algorithms; the method comprised calculating a three- dimensional Euclidean distance map, clustering voxels, and extracting the network of the pore space. They also described partitioning the pore space into nodes and bonds and determining geometrical features, such as radii and shape factors. Prodanović et al. (2007) studied fluid displacement in a Berea core sample, and used MCT to obtain three-dimensional images of the pore structure. The highresolution images provided detailed pore geometry characteristics (e.g., pore volume, and throat area) as input to Lattice-Boltzmann models, which were used to relate porescale parameters to bulk flow properties (e.g., absolute permeability).

Properties such as residual trapping, interfacial area, and capillary forces have also been defined based on highresolution images from synchrotron X-ray CT. Culligan et al. (2006) suggested that quantifying the nonaqueous phase liquid-water interfacial area provides a measure of the expected area available for mass transfer dissolution in porous media. Al-Raoush and Willson (2005) used synchrotron X-ray tomographic images of a multiphase porous media system to capture the entrapment of the nonwetting phase at residual saturation. This study provided a framework for using high-resolution three-dimensional imaging to extract the pore network and quantify fluid distributions. The results showed the non-wetting phase trapped primarily in the largest pore spaces and in the pore bodies with the highest aspect ratios. In addition, the use of highresolution X-ray CT provided datasets of a sufficiently high quality to observe the representative elementary volume. However, the size of these images was not sufficient to provide estimates of some of the bulk properties, such as residual saturations and interfacial areas.

Previous investigations of trapped oil clusters in waterwet glass bead packs using X-ray microCT by Karpyn et al. (2010) were performed at larger dimensions (approximately $25.4 \mathrm{~mm}$ in diameter and $90.53 \mathrm{~mm}$ long). The granular system used in this experiment consisted of spherical glass beads ranging from 0.40 to $0.60 \mathrm{~mm}$ in diameter. A spatial distribution of the trapped oil clusters for the entire bead pack was presented to explain fluid distribution. Likewise, Landry et al. (2011) presented quantitative evidence of immiscible fluid distribution in an oil-wet acrylic bead pack, thereby offering a direct comparison with the results presented by Karpyn et al. (2010). Landry's experimental results showed how wettability and saturation history affect fluid distribution. In addition, it was found that the specific surface area of the wetting phase of the acrylic bead pack was less than for the glass bead pack, whereas the specific area of the nonwetting phase of the acrylic bead pack was greater than that of the glass bead pack. Thus, suggesting that the affinity of acrylic beads- to oil was weaker than that of glass beads to water. 
With the notable exceptions of Lebedeva and Fogden (2011), Delshad et al. (2006) and Anderson (1987), the vast majority of the work found in the literature focuses on uniformly wetted systems (e.g., water-wet and oil-wet systems). However, it is generally accepted that most geologic porous media are not uniformly wetted. In addition, crude oil can alter the rock state by adsorbing or depositing polar components, thus resulting in a mixed-wet state of relatively oil-wet subareas where oil makes contact combined with unaltered subareas covered by brine. The term "mixed-wettability" was introduced by Salathiel (1973) to describe systems with large pores that are predominantly oil-wet and smaller ones that are water-wet, and in which the residual oil saturation is much lower than that obtained in uniformly wetted systems. Donaldson et al. (1969) reported that changes in wettability affect water flood behavior. They treated the core samples with various amounts of organochlorosilane to change the wettability of the cores from water-wet to oil-wet. The results showed that less oil was recovered after a water injection as the system became more oil-wet. Moreover, according to Morrow Norman and Geoffrey (2001) an infinite number of possible wetting states exist between the strongly waterwet and the strongly oil-wet, and similarly there are many varied flow behaviors Tiab and Donaldson (2004). Understanding the effect of wettability on fluid recovery requires information pertaining to pore-scale fluid distribution, wetting preferences, and pore geometry Kumar (2008). Multiple pore-level models have been proposed to explain the effect of mixed wettability. Heiba et al. (1983) used network models to study the distribution of oil under mixed-wettability conditions. Raeesi and Piri (2009) used a pore-scale network model to study the effect of wettability and trapping. Radke et al. (1992) developed a pore-level illustration to explain how mixed-wettability forms and how it affects the reservoir rock.

The purpose of this study is to analyze a mixed wettability granular pack, and to provide a comparative framework for previous uniform wettability experiments reported in the literature, focusing on the interplay of porosity distribution, fluid trapping behavior, and the effect of wettability. The study offers comprehensive information about the pore structure and immiscible fluid spatial distribution. Such information can be used to generate a basis for porelevel modeling and macroscopic-level predictions. A bead pack with a mixture of glass and acrylic beads was used to represent mixed wettability conditions. The dimensions of the system are representative to those of previous experiments reported in the literature. The results were obtained using the following approach: MCT was used to map the residual fluids (oil, water) and the solid beads (glass, plastic); the resulting image sets were segmented to identify the plastic and glass beads, the oil phase, and water phase. From these data, the porosity, fluid blob size, and saturation distributions were obtained. Finally, the obtained results for the mixed wettability system were compared to uniform wettability data.

\section{Methodology}

Summary of data set protocol

For the mixed wettability system, glass and plastic beads, approximately $0.40-0.60 \mathrm{~mm}$ in diameter, were packed into a cylindrical plastic core holder of $103.32 \mathrm{~mm}$ in length with an inner diameter of $25.4 \mathrm{~mm}$. The proportion of glass and plastic beads in the mix was 66.61 and $33.39 \%$, respectively.

Flow distributors were added to the upper and lower ends of the bead pack to achieve a uniform flow profile. In addition, a metal screen was placed between the flow distributors and the bead pack. The core holder was placed in the X-ray microCT scanner in the vertical position.

The dry bead pack was first evacuated and pre- saturated with brine. Kerosene was then injected into the pack to achieve irreducible water saturation, and finally brine was injected into the pack until residual oil saturation was achieved. The lighter phase, kerosene was injected from the top of the core holder to minimize buoyancy effects, whereas the heavier brine phase was injected from the bottom to minimize gravitational segregation due to the density difference of the fluids. At the end of each stage of fluid injection, the core sample was scanned using MCT. The experimental sequence is shown in Fig. 1.

In this project, MCT was used to determine the fluid structures in the porous medium. The MCT system consists of an X-ray source, a detector, a translation system, and a computer system that controls motion and data acquisition Karpyn et al. (2010). MCT imaging is a visualization technique used for examining internal structure of opaque three-dimensional objects by producing stacks of twodimensional images (slices) that reveal the interior of the objects in a nondestructive manner Denison et al. (1997). The scans of this core sample were composed of 3,900 slices, each of which consisted of $1,024 \times 1,024$ pixels. The MCT system was tuned to a voxel resolution of $0.026 \times 0.026 \times 0.027 \mathrm{~mm}$, in which $0.027 \mathrm{~mm}$ represented the height of the voxel or the thickness of each slice.

\section{Data sets}

Four sets of images were obtained using MCT at different stages: dry sample, $100 \%$ brine saturated sample, irreducible brine saturation $\left(S_{\text {wirr }}\right)$, and residual oil saturation $\left(S_{\text {or }}\right)$. 
Fig. 1 Schematic diagram of the experimental procedure showing the sequence of the kerosene (oil) and brine (water) injections

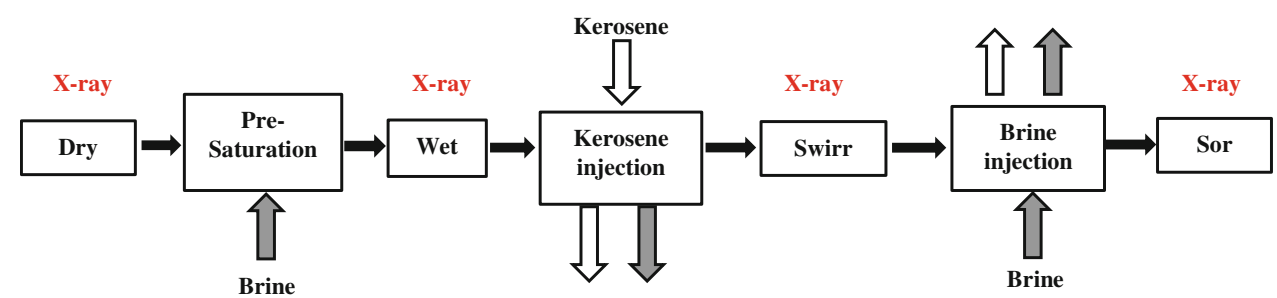

\section{Dry beads}

The vacuumed bead pack was scanned dry to obtain an accurate map of the beads and pore structure. Differences in CT numbers for beads and pore space is large due to the difference in density and atomic number. High-density materials characteristically have a high CT number (represented by the light gray regions), whereas low-density materials characteristically have a low CT number (represented by the dark gray regions). Therefore, at this stage, it was easy to segregate the beads from the pore structure.

\section{Wet beads}

After the dry sample was scanned, the core sample was presaturated with brine and scanned again. The resulting images were used in the segmentation process to obtain the fluid distribution in the pore space. Compared with the plastic bead, the glass beads and the brine are denser and are shown in light gray, whereas the plastic beads are shown in dark gray in the images.

\section{Irreducible water saturation $\left(S_{\text {wirr }}\right)$}

At this stage, kerosene was injected into the core sample to displace the brine, until it reached irreducible water saturation. Then, the core sample was scanned. For this stage, most of the pore space was filled with kerosene with only a small amount of brine. As the plastic beads and the kerosene are less dense than the glass beads and brine, the regions occupied by kerosene and plastic beads are darker than the regions occupied by glass beads and brine.

\section{Residual oil saturation $\left(S_{\text {or }}\right)$}

The last stage was to inject brine into the core sample to displace the fluid in the previous stage, until it reached residual oil saturation. Then, the core sample was scanned. In contrast to the previous stage, most of the pore space was filled with brine with only a small amount of kerosene. Again, as the plastic beads and the kerosene are less dense than the glass beads and brine, the regions occupied by glass beads and brine are brighter than the regions occupied by kerosene and plastic beads. Thus, the major difference between $S_{\text {wirr }}$ and $S_{\text {or }}$ is that the pore space in $S_{\text {wirr }}$ appears to be dark (dark gray), whereas the pore space in $S_{\text {or }}$ appears to be bright (light gray).

\section{Segmentation and quantification}

In image processing, the most important step is to separate two distinct materials from each other, i.e., foreground (object) and background, using a proper thresholding method. The simplest segmentation techniques employ global thresholding in which one threshold value is applied to the whole system. Values below the threshold value are designated as one material, and values above are designated to the other material Al-Raoush and Willson (2005).

Various techniques have been used to obtain the most accurate threshold value, such as k-means cluster analysis used by Porter and Wildenschild (2010); local thresholding criteria based on indicator kriging mentioned in Prodanović et al. (2007); and the Otsu method used by Otsu (1979).

Additionally, Mehmet (2004) reported a survey of over 40 image-thresholding techniques to categorize and compare the performance of each. The thresholding techniques were categorized into six major groups according to the information explored such as histogram-shape-based methods, clustering-based methods, entropy-based methods, object-attribute-based methods, spatial methods, and local methods. The performance criteria were based on the misclassification error, edge mismatch, relative foreground area error, modified Hausdorff distance, and region nonuniformity. The inclusive details are described in the survey of image-thresholding techniques and quantitative performance evaluation (2004). The final evaluation performance scores of each method were calculated and ranked from highest to lowest. For the nondestructive testing image datasets, clustering-based methods are among the seven highest-ranking techniques.

One of the most effective clustering-based methods is the Otsu thresholding, which works by iterating between all the possible threshold values to find a particular value where the sum of the foreground and the background is at its minimum. Assume that the number of pixels at the gray level $i$ from all the L levels is denoted by $n_{i}$ and the number of pixels $(N)$ is the summation of $n_{i}$, which is 
$N=n_{1}+n_{2}+n_{3} \ldots . n_{L}$. The gray level probability distribution $\left(p_{i}\right)$ is

$p_{i}=\frac{n_{i}}{N}$

At threshold level $k$, the pixels are divided into two classes: $\quad C_{0}$ [background, $\left.(1,2, \ldots, k)\right]$ and $C_{1}$ [foreground, $(k+1, \quad k+2, \ldots, \quad L)$ ]. Thus, the probability, mean, and class variances for each class can be described as follows:

Class $C_{0}$ :

Background probability

$\omega_{0}=\sum_{i=1}^{k} p_{i}$

Background mean value

$\mu_{0}=\sum_{i=1}^{k} \frac{i p_{i}}{\omega_{0}}$

Background class variance

$\sigma_{0}^{2}=\sum_{i=1}^{k} \frac{\left(i-\mu_{0}\right)^{2} p_{i}}{\omega_{0}}$

Class $C_{1}$ :

Foreground probability

$\omega_{1}=\sum_{i=k+1}^{L} p_{i}$

Foreground mean value

$\mu_{1}=\sum_{i=k+1}^{L} \frac{i p_{i}}{\omega_{1}}$

Foreground class variance

$\sigma_{1}^{2}=\sum_{i=k+1}^{L} \frac{\left(i-\mu_{1}\right)^{2} p_{i}}{\omega_{1}}$

where the total mean is

$\mu_{T}=\sum_{i=1}^{L} i p_{i}$

To evaluate the correctness of the threshold value, three parameters are calculated,

$\lambda=\frac{\sigma_{\mathrm{B}}^{2}}{\sigma_{\mathrm{W}}^{2}}, \kappa=\frac{\sigma_{T}^{2}}{\sigma_{\mathrm{W}}^{2}}, \eta=\frac{\sigma_{\mathrm{B}}^{2}}{\sigma_{T}^{2}}$,

for within-class variance:

$\sigma_{\mathrm{W}}^{2}=\omega_{0} \sigma_{0}^{2}+\omega_{1} \sigma_{1}^{2}$

between-class variance:
$\sigma_{\mathrm{B}}^{2}=\omega_{0}\left(\mu_{0}-\mu_{T}\right)^{2}+\omega_{1}\left(\mu_{1}-\mu_{T}\right)^{2}$

and, total variance:

$\sigma_{T}^{2}=\sum_{i=1}^{L}\left(i-\mu_{T}\right)^{2} p_{i}$

To obtain an accurate threshold value, these three parameters must be optimized. In other words, the threshold that provides the minimum within-class variance $\left(\sigma_{\mathrm{w}}^{2}\right)$ and the maximum between-class variance $\left(\sigma_{\mathrm{B}}^{2}\right)$ will be the best threshold value.

In this study, the Otsu method was implemented via the graythresh algorithm in MATLAB. Each pixel at the different gray level $i$ was counted and stored to construct a histogram, and the number $i$ was used as the number of bin. The advantages of this method are its implementation simplicity; its stability is based on the integration of the histogram, and it covers a wide scope of unsupervised decision procedures.

This method can be applied to separate only two different materials at a time. To segment the three-phase or four-phase systems using Otsu thresholding, a two-step approach is applied. First, the pore space is segmented from the solid (glass and plastic) beads by subtracting the dry sample from the wet sample to remove the solid beads. Thus, the two-phase system is obtained. Then, the graythresh MATLAB algorithm was applied again to the extracted sample set, such that all the voxels with a CT number lower than the thresholding value $(k)$ represented the subtracted solid and all the voxels with a CT number higher than the thresholding value $(k)$ represented the pore space (higher CT number). Once the pore space and the subtracted solid space have been segmented, their discretized $3 \mathrm{D}$ grids are used to remove the bead space or pore space from the subsequent core sample scans. The next step was to segment the kerosene from the brine and to segment the glass beads from the plastic beads. The 3D grid of the pore space binary 1 and the $3 \mathrm{D}$ grid of the solid space binary 2 images were multiplied to the $S_{\text {wirr }}$ or $S_{\text {or }}$, and dry sample sets to isolate the fluid in the pore space area, and the mixed beads in the solid area, respectively. Similarly, the graythresh algorithm was applied to the multiplied samples to obtain their thresholding values. Once the residual oil and water were segmented from the $S_{\text {or }}$ and $S_{\text {wirr }}$ samples, respectively, their volume and the surface area for each of the disconnected blobs were analyzed using the three-dimensional visualization software Avizo 3D version 6.3. The stack of images was processed to obtain volume and surface area individually for each separate body. The volume of each segmented body, $X$, was approximated by the number of voxels forming it: 
$V(X)=\sum_{i, j, k} I\left(x_{i}, y_{j}, z_{k}\right)$,

where $I\left(x_{i}, y_{j}, z_{k}\right)$ is the intensity of the pixels of the coordinates $x_{i}, y_{j}$, and $z_{k}$. The volume of the pixels was scaled to give the true area in the unit associated with the image. Note that, when applying this to binary images, $I$ is equal to 1 , or 0 . Moreover, the application area 3D was approximated using the intercept, defined as the number of entries in an object along a given direction to account for the exposed surface of the outer pixels.

In addition to the quantification of the fluid saturation, the residual fluid volume, and the surface area, the explicit imaging of the plastic and glass beads was used to evaluate the proximity of the residual fluid structure to the define solid surface via MATLAB. This was achieved by computing the shortest distance from each fluid pixel (blob) to a particular solid type (plastic or glass) to describe the tendency of the fluid trapping with respect to wettability. To determine the shortest distance, the boundary pixels were first defined for both the blobs and the beads by considering the closest 26 neighboring pixels to each. Any pixels surrounded by 26 neighboring pixels were not counted as boundary pixels. The distance between the fluid blob pixels and any solid bead pixel was calculated using the Euclidian distance $\left(D_{\mathrm{E}}\right)$ :

$D_{\mathrm{E}}=\sqrt{\left[\left(\left(X_{\mathrm{s}}-X_{\mathrm{f}}\right) \times x\right)^{2}+\left(\left(Y_{\mathrm{S}}-Y_{\mathrm{f}}\right) \times y\right)^{2}+\left(\left(Z_{\mathrm{s}}-Z_{\mathrm{f}}\right) \times z\right)^{2}\right]}$

where $X_{\mathrm{s}}, Y_{\mathrm{s}}$, and $Z_{\mathrm{s}}$ represent the solid pixel coordinates; $X_{\mathrm{f}}, Y_{\mathrm{f}}$, and $Z_{\mathrm{f}}$ represent the fluid pixel coordinates; and $x, y$, and $z$ represent the voxel size of $x, y$, and $z$ directions, respectively. Once a fluid voxel was found, then the shortest distance to a solid surface is computed. In this analysis, 800 slices were considered in the mid-region, which was approximately one-third of the core sample.

\section{Results and discussion}

\section{Porosity distribution}

The average porosity of the bead pack was found to be $42.04 \%$, with local axial porosity values at different heights along the $z$-axis varying from 39.99 to $44.55 \%$, as shown in Fig. 2. The average porosity is in agreement with the theoretical porosity for rhombohedral and cubic packing of regular-sphere grains, which is 25.96 and $47.64 \%$, respectively. This suggests that the packing is mostly cubic. Moreover, a small variation in percent porosity is good evidence that the bead packing is relatively homogeneous, with the greatest variation at $10 \mathrm{~mm}$ in height.

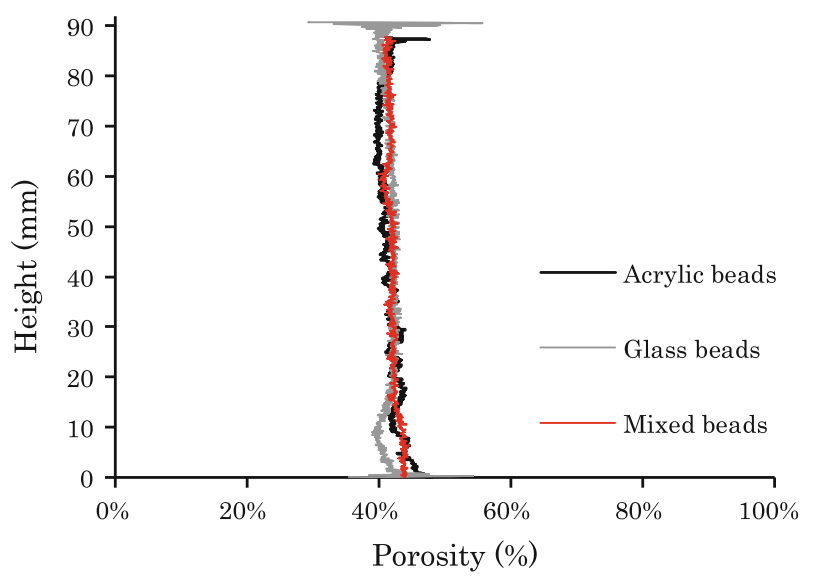

Fig. 2 Vertical profile of bead pack porosity

The average porosity for the mixed-bead pack is comparable to that of the glass bead and plastic bead packs where their average porosity was 41.6 and $42.1 \%$, respectively. All of the three bead packs show a drop in porosity at the bottom part, at $20 \mathrm{~mm}$ in height that may have resulted from localized compaction.

Fluid phase distribution

In addition to pore space and solid region segmentation, each fluid phase within the pore space at $S_{\text {wirr }}$ and $S_{\text {or }}$ was defined. The segmentation procedure was similar to that used for the previous two sample sets.

For $S_{\text {wirr }}$, kerosene was injected to displace the brine from the pore space, resulting in a small proportion of brine being trapped inside the pore space (irreducible water), whereas the rest of the pore space was filled with kerosene. For $S_{\text {or }}$, brine was injected to displace all the mobile kerosene from the previous stage, resulting in a small proportion of kerosene being trapped inside the pore space (residual oil). The rest of the pore space was filled with brine.

Trapped fluid blobs segmented from CT data ranged in size from $1.9 \times 10^{-5} \mathrm{~mm}^{3}$ (approximately one single voxel) to $1.18 \times 10^{3} \mathrm{~mm}^{3}$ for water blobs, and from $1.9 \times 10^{-5}$ to $1.95 \times 10^{3} \mathrm{~mm}^{3}$ for oil blobs. The total volume of the water blobs and the oil blobs was $2.7 \times 10^{3}$ and $3.1 \times 10^{3} \mathrm{~mm}^{3}$, respectively. Moreover, the single largest oil blob found at residual oil saturation was larger than any trapped water blob, suggesting that water remained trapped at the crevices of grain-to-grain contacts and therefore a more dominant wetting phase in this mixedwet system.

The blob cumulative frequency in Fig. 3 shows that most of the blobs fell in a size between 0.0001 and $0.001 \mathrm{~mm}^{3}$, making them 100 times smaller in size than those in the single-wettability glass and plastic bead packs 
Fig. 3 Cumulative water blob frequency after the oil injection and oil blob frequency after the water injection

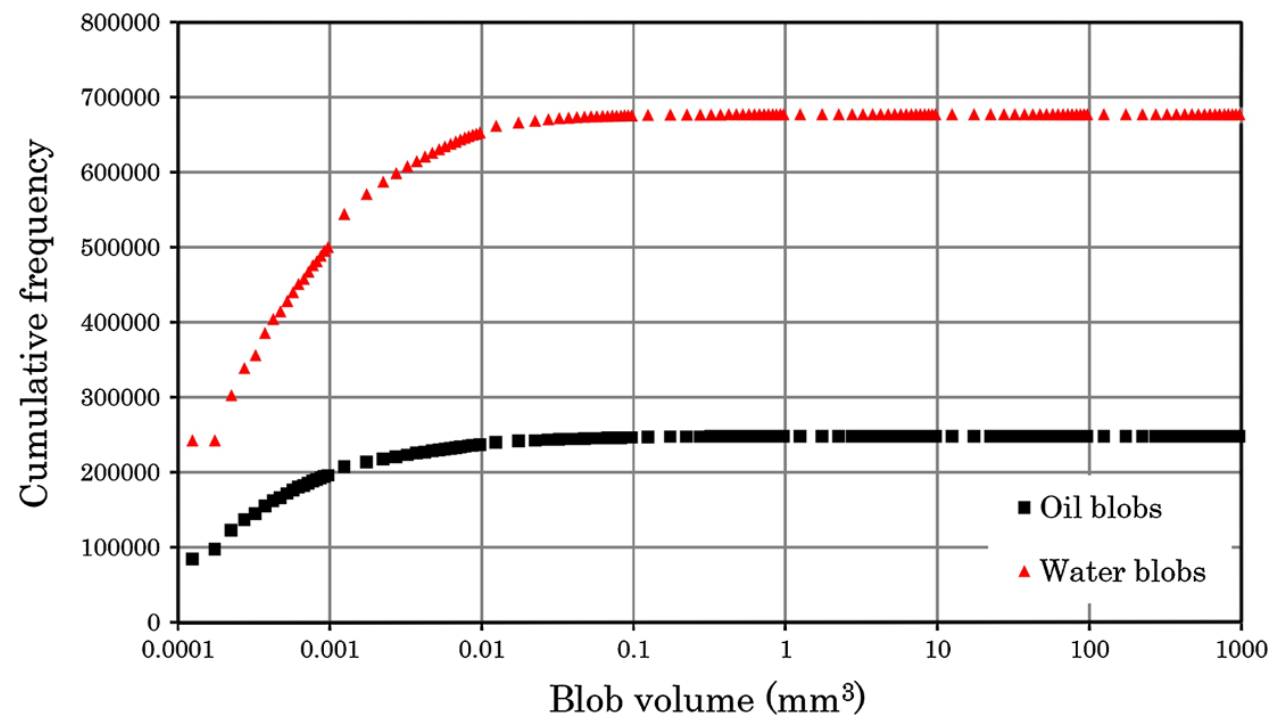

reported by Karpyn et al. (2010) and Landry et al. (2011). This difference in sizes of the blobs is expected since the wetting fluid tends to be imbibed by the small pores in the uniformly wet system, causing the nonwetting fluid to be trapped in the larger pores Raeesi and Piri (2009), Kumar et al. (2008), Zhao et al. (2010) and Al-Futaisi and Patzek (2003).

Moreover, Fig. 3 demonstrates a larger quantity of small water blobs as compared to oil blobs. This observation can be explained by the higher proportion of glass beads (water-wet surfaces) compared to acrylic beads (oil-wet surfaces), given that water will remain trapped in the pendular rings around the water-wet grain contacts. Interesting analogies from natural systems can be found in Tiab and Donaldson (2004), Sahimi (1995) and Kumar et al. (2008), whereby water blobs formed from the pre- saturation of brine, as the small water-wet pores are spontaneously imbibed by water due to capillary suction.

Figure 4 shows the cumulative volume percent of each oil blob group to the total residual oil trapped in the pore space and each water blob group to the total residual water trapped in the pore space. Disregarding the largest blob volume, the steepest slope of the oil blobs plot and the water blobs plot was found in the range of $0.01-1 \mathrm{~mm}^{3}$ and $0.001-0.1 \mathrm{~mm}^{3}$, respectively, suggesting that the blobs in these groups account for an important portion of the total residual fluid trapped in the pore space. This examination is consistence with the findings of Salathiel (1973), according to which smaller pores are filled with water, whereas the oil preferentially wets larger interconnected pores Anderson (1987).

The spatial distribution along the $z$-direction of all the disconnected water blobs and oil blobs is presented in
Fig. 4 Cumulative volume percent of water blob group to the total residual water and oil blob group to the total residual oil

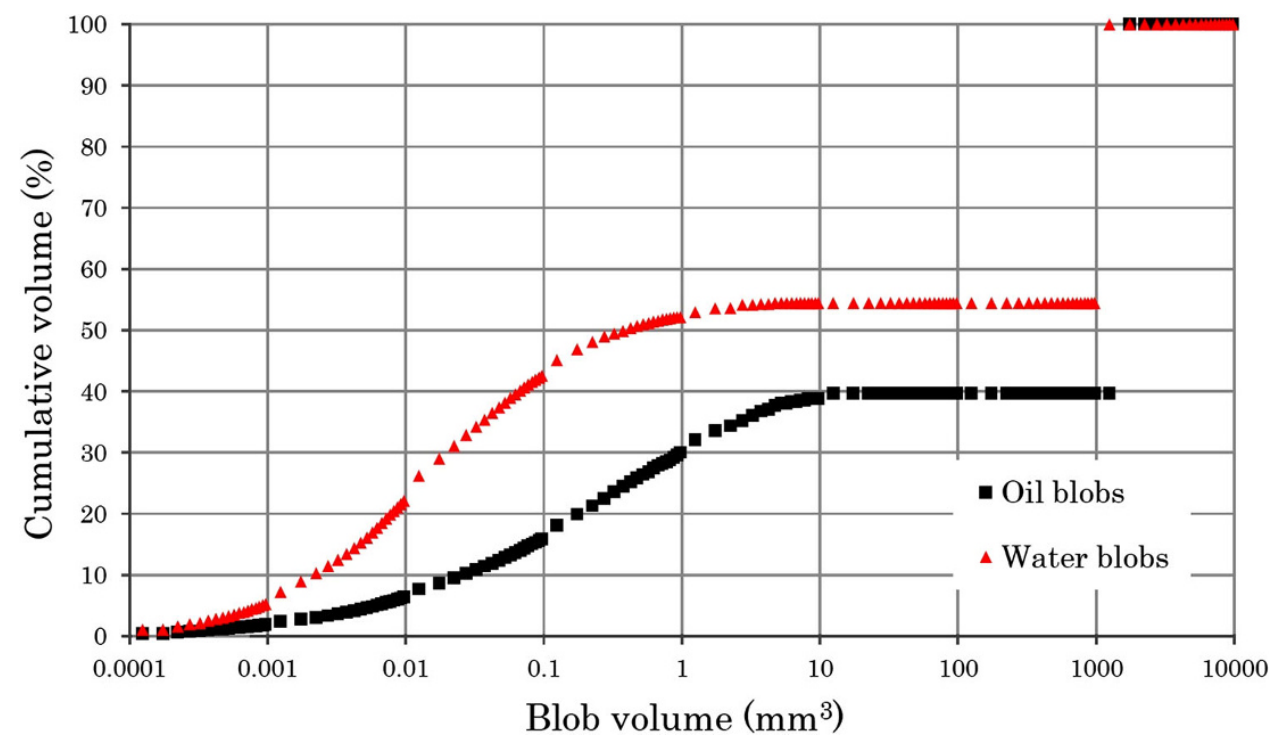

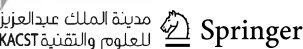




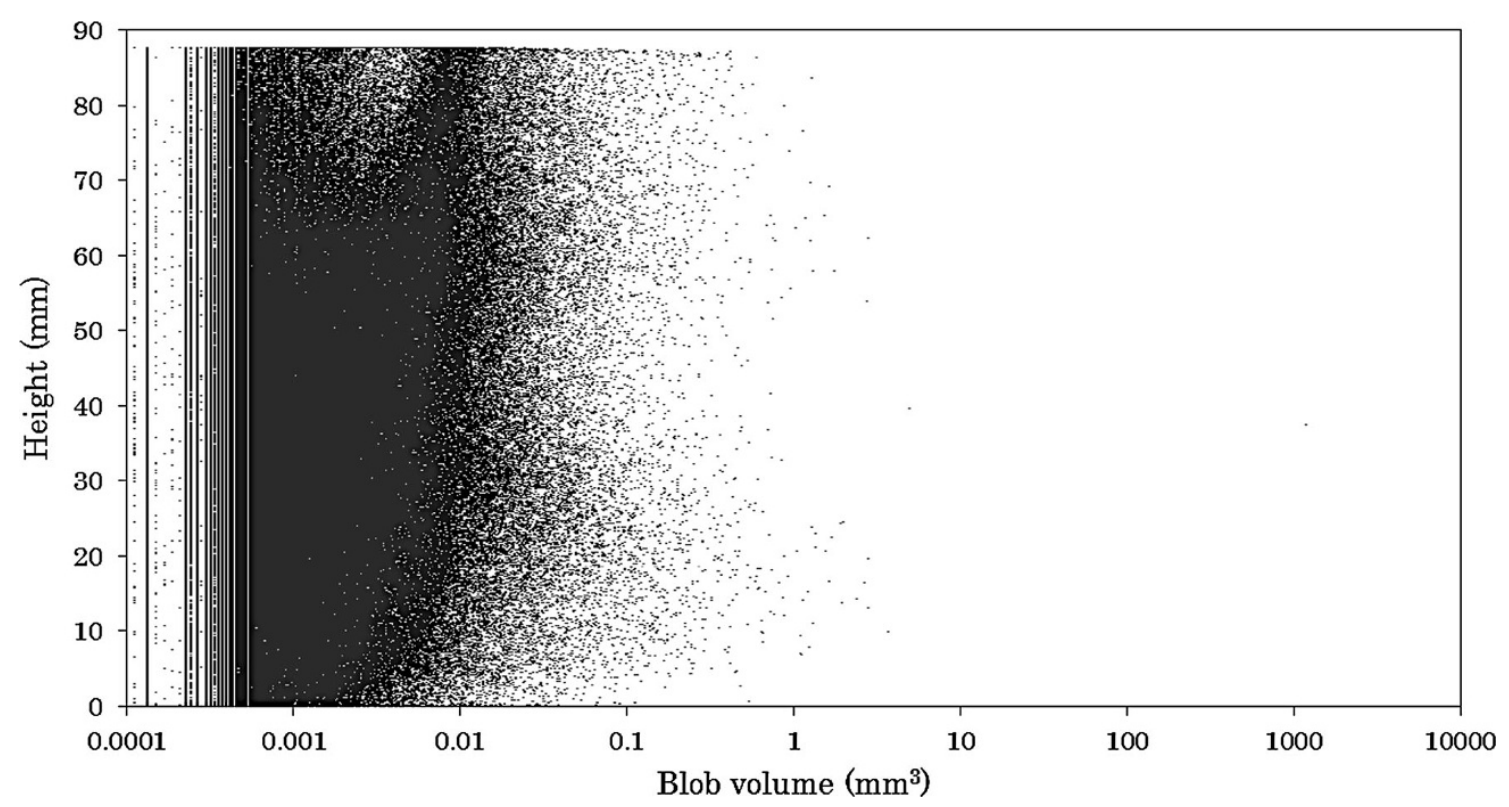

Fig. 5 The spatial distribution along the $z$-direction of the disconnected water blobs after oil injection

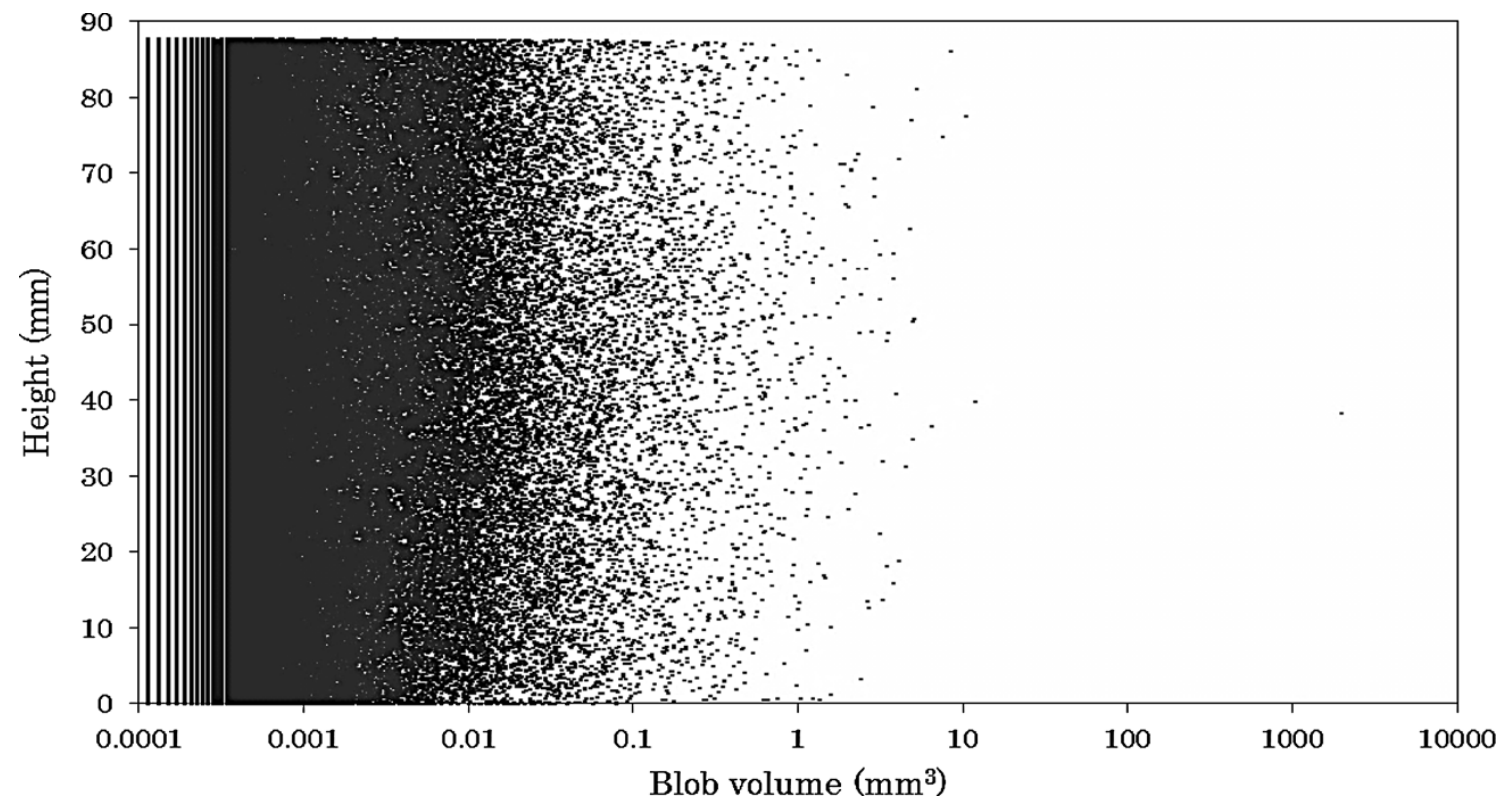

Fig. 6 The spatial distribution along the $z$-direction of the disconnected oil blobs after water injection

Figs. 5 and 6, respectively, wherein each spot represents the vertical location of the centroid of each blob. In the water blob distribution plot, there are smaller amount of medium size blobs on the upper parts and smaller amount of large size blobs on the lower parts of the bead pack. With regard to oil blob distribution, the plot shows that the oil blobs were distributed homogeneously throughout the bead packs. This suggests that oil blobs size is more uniform than that of water blobs.
Individual water blob volumes and oil blob volumes were plotted against their surface area in which each spot in the plot represents one disconnected blob, as shown in Fig. 7. The surface to volume ratio of a sphere was also plotted using a solid line as a reference. The plots show that the blobs were located close to the reference line when their volume is small, and then start to deviate from the reference line as their volume increases. The deviation started at a volume of $0.02-0.03 \mathrm{~mm}^{3}$, which is 


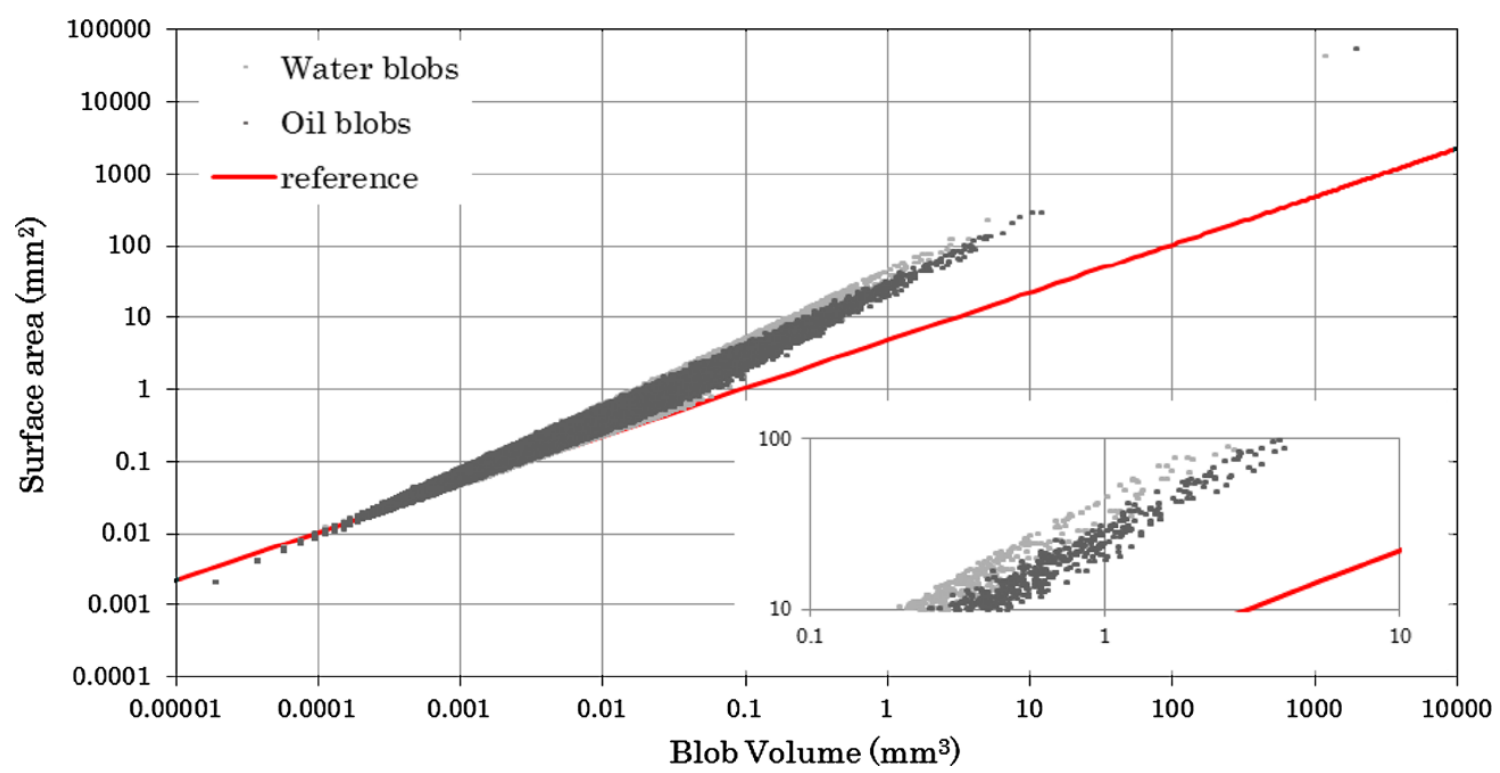

Fig. 7 Water blob surface area as function of blob volume after oil injection and oil blob surface area as a function of blob volume after water injection

Table 1 Average percent water saturation

\begin{tabular}{lcc}
\hline Pore system & End of oil injection (\%) & End of water injection \\
\hline Mixed-wet & 14.08 & $83.61 \%(16.39 \%$ oil $)$ \\
Oil-wet & 8.32 & $76.14 \%(23.86 \%$ oil $)$ \\
Water-wet & - & $82.28 \%(17.72 \%$ oil $)$ \\
\hline
\end{tabular}

comparable to the mean pore volume. At volumes larger than $0.03 \mathrm{~mm}^{3}$, the blobs no longer behave like spheres and start to extend from a pore through other interconnected pores. The porosity was found to be similar to that reported by Karpyn et al. (2010), given the grain size distribution and packing was almost the same in both samples. The largest blob volume, that of $10^{3} \mathrm{~mm}^{3}$, has the largest surface to volume ratio and was expected to have the highest extension to other interconnected pores. For the small blob volume, as these blobs were close to the reference line, their shape was likely to be almost spherical. The trend of surface area to blob volume for both oil and water was very similar, with a slight tendency of water blobs to reach a higher surface area for the same blob volume which can be attributed to stronger water wetting affinity to the solid surface than that of oil.

\section{Percent water saturation}

The average percent water saturation after the oil injection and the water injection was shown in Table 1. As noted earlier, the bead pack was saturated with water (brine) followed by the oil (kerosene) injection from the top until the amount of water collected at the outlet of the core was negligible, which meant that the water trapped inside the bead pack was at irreducible water saturation. The amount of trapped water was measured per slice and was averaged across the vertical direction. The average water saturation was found to be $14.08 \%$ and the rest of the pore space was filled with oil (i.e., at $85.92 \%$ ).

In comparative analysis with findings reported by Landry et al. (2011) for acrylic bead packs, average oil saturation after the first imbibition (oil injection process) was reported to be $91.68 \%$ such that only $8.32 \%$ of the water was trapped. This behavior was expected, as acrylic beads are oilwet; therefore, an interface force between the oil and the acrylic beads was stronger than that between the oil and glass. And given that oil is more likely to attach to acrylic beads, the result was higher percent oil saturation. This observation also corresponds with findings reported by Jadhunandan and Morrow (1995) on the relationship between wettability and irreducible water saturation in which water-wet rock has a water saturation of greater than 20-25\% whereas oil-wet rock commonly has a water saturation of less than $15 \%$ of pore volume and frequently less than $10 \%$.

Moreover, McCaffery and Bennion (1974) found that irreducible water saturation tends to decrease with increasing oil wettability. The observation is based on the fact that in water-wet rocks, water covers the pore surfaces and thus exists as a continuous film; however, in oil-wet rocks in which the solid grains are mostly covered by oil, residual water is likely to behave like disconnected blobs in the center of the pore space Anderson (1987) and Dandekar (2006). 
In the next stage, brine was injected into the core sample until the amount of oil collected at the outlet of the core was negligible, indicating that the oil phase was at the residual oil saturation. The average residual oil trapped was found to be $16.39 \%$ and the rest of the pore space was filled with brine (i.e., at $83.61 \%$ ). Compared to related findings reported by Landry et al. (2011) for acrylic bead packs and by Karpyn et al. (2010) for glass bead packs, the average residual oil saturation after water injection was reported to be 23.86 and $17.72 \%$, respectively. In the case of a uniformly wet system (e.g., the water-wet system), the wetting fluid brine tends to form a continuous thin film over the solid surface, and to fill in small or medium pores while displacing the oil into the larger pores.

This results in the oil occupying the center of the larger pores such that continuous channels of oil are formed or oil becomes trapped as disconnected blobs. At slow flow rates, the water moving in a fairly uniform front, displaces oil by a piston-like mechanism (as the capillary pressure is greater than the threshold pressure), and by spontaneous imbibition (where capillary forces make the water phase spontaneously displace the oil from small or medium pores) Patzek (2001), Kumar et al. (2008), Wilkinson and Willemsen (1983) and Wardlaw (1982). As the water bypasses the oil, it swells (due to the decrease in capillary pressure) and breaks down the oil, forcing it into the large pores. This process is referred to as "snap-off" Lenormand et al. (1983), Oren et al. (1997) and Patzek (2001). For oilwet systems, most of the oil in the large pores is recovered, whereas the remainder is trapped in the smaller pores due to capillary forces. And, as these pores are oil-wet, there is no driving force to cause the water to displace the oil from them.

Thus, the mixed-wet system significantly trapped smaller amount of residual oil compared to oil-wet system, which is consistent with Amott's finding that extreme wettability results in high residual oil, whereas weakly water-wet conditions or slightly oil-wet conditions result in low residual oil. It may be that the mixed wet system trapped less residual oil as a result of the oil-wet solid surface that allows the oil blobs that are close to each other to connect and form a continuous flow path. As such, an oil-wet solid surface prevents water from forming a continuous thin film, and prevents snap-off. Likewise, the oil trapped in the smaller pores is reduced as compared to an oil-wet system, because the small pores in a mixed-wettability system are water-filled Anderson (1987). According to Anderson (1987), as compared to the water-wet system, the mixed-wet system traps less oil because it traps less oil in the larger oil-wet pores. However, in this experiment, there was no significant difference between the percent oil trapping in mixed-wet $(16.39 \%)$ and water-wet systems $(17.72 \%)$. The residual oil was as small as that of
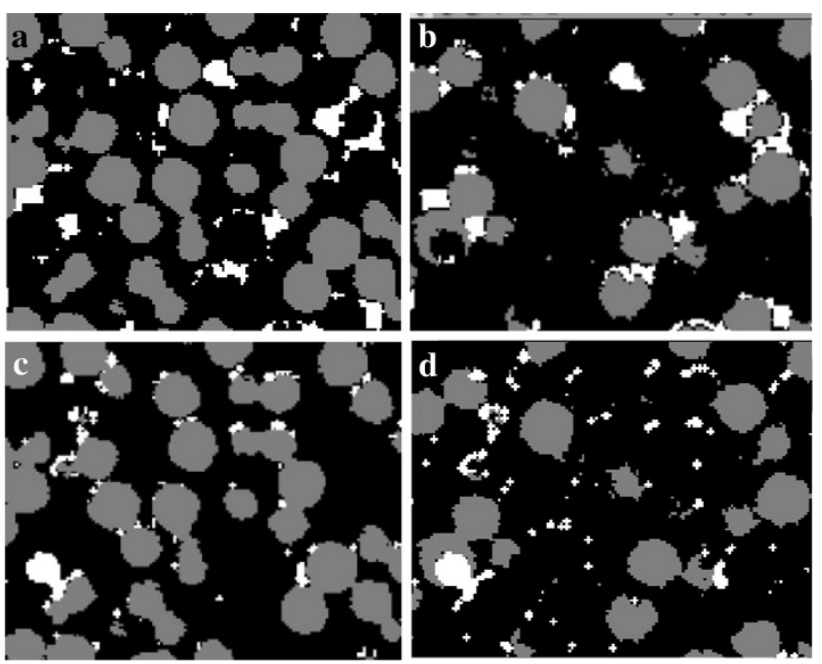

Fig. 8 Residual fluid structures (in white) and selected solid material (in gray) a oil and glass beads; b oil and plastic beads; c water and glass beads; d water and plastic beads

the water-wet system or perhaps even smaller, suggesting that global phase trapping in porous media (residual saturation) is sensitive to the wetting characteristics of the solid matrix. Moreover, the saturation history of oil might affect the amount of residual oil trapped after the water injection, as the amount of oil saturation of the acrylic bead packs $(91.68 \%)$ was higher than that of mixed-bead packs $(85.92 \%)$, resulting in higher residual oil saturation.

However, the change in residual oil saturation is small, whereas wettability ranges from one extreme to the other. Masalmeh (2003) also reported that in mixed-wet conditions, the small pore and grain contacts are preferentially water-wet but that the surfaces of the larger pores are oilwet. As oil-wet paths were continuous through the larger pores, water could displace oil from the pores and very little oil would be held by capillary forces in small pores or at grain contacts. This resulted in low residual oil and high oil recovery, as reported by Salathiel (1973), and Anderson (1987).

\section{Wetting affinity}

To evaluate the wetting affinity of the glass and plastic beads, we examined the proximity of residual fluid structures to each solid surface (plastic and glass). Figure 8 displays sections of the mixed-bead pack relative to residual fluid structures. Figure 8 a represented the residual oil structures and the glass beads whereas Fig. 8b represented the residual oil structures and the plastic beads. The results showed that the positions of the residual oil were closer to the plastic beads than to the glass beads such that we observed the plastic beads surrounded by the residual oil structures. In contrast to the residual oil, the positions of 
the residual water were closer to the glass beads than the plastic beads as shown in Fig. 8c, d, respectively, and we observed the glass bead surrounded by the residual water structures.

In addition, the preferential affinity of each fluid type to a particular solid surface was quantified by calculating the shortest distance between fluid and solid surfaces. The frequency distributions of the shortest distance were divided into 50 bins and plotted. The shortest measurable distance was $0.026 \mathrm{~mm}$, given by the imaging resolution, which means that the pixel of the fluid blob is next to the pixel of the solid bead. In general, the shorter the distance between the fluid and the solid beads, the greater is the wetting affinity of the fluid to a particular solid; i.e., the more likely the fluid is to come into contact with and spread over or adhere to a solid surface. Results of this study are presented in Figs. 9 and 10. Figure 9 reveals a narrower distribution of oil-to-plastic distances than that of oil-to-glass. This is evidence that the plastic beads are preferentially oil-wet relative to the glass beads. A similar analysis of Fig. 10 confirms that glass beads are more water-wet than the plastic beads, and that the wetting affinity of the glass beads with regard to water was stronger than that of the plastic beads.

\section{Conclusions}

Immiscible fluid flow through a synthetic bead pack with mixed wettability was investigated. Three-dimensional imaging of this bead pack after cycles of oil and water injections allowed the characterization of the final saturation distribution and of the trapped fluid structures in the context of surface wettability. In addition, results were compared against previous experimental findings in a uniformly water-wet glass bead pack and oil-wet plastic bead pack. The average porosity of the mixed-wet system was $42.04 \%$, which is comparable to that of water-wet and oil-wet systems at 41.6 and $42.1 \%$, respectively, suggesting that the packing was mostly cubic.
Fig. 9 Histogram of the shortest distance from the oil blobs to the plastic beads and the shortest distance from the oil blobs to the glass beads

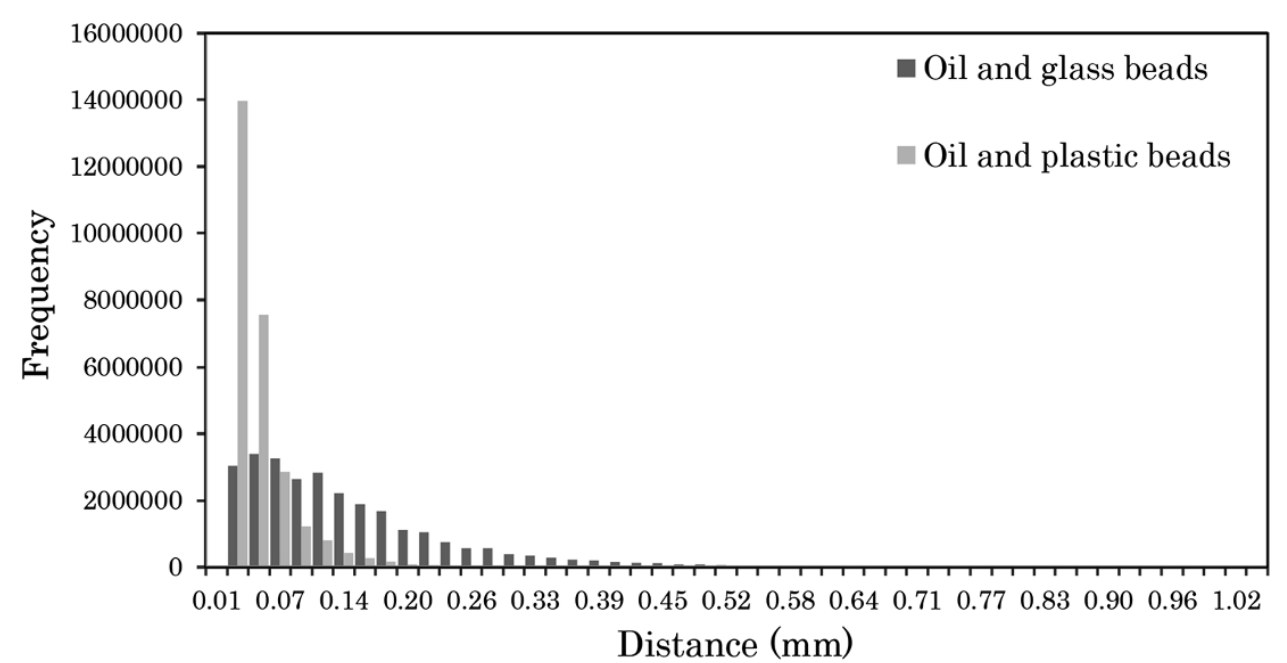

Fig. 10 Histogram of the shortest distance from the water blobs to the plastic beads and the shortest distance from the water blobs to the glass beads

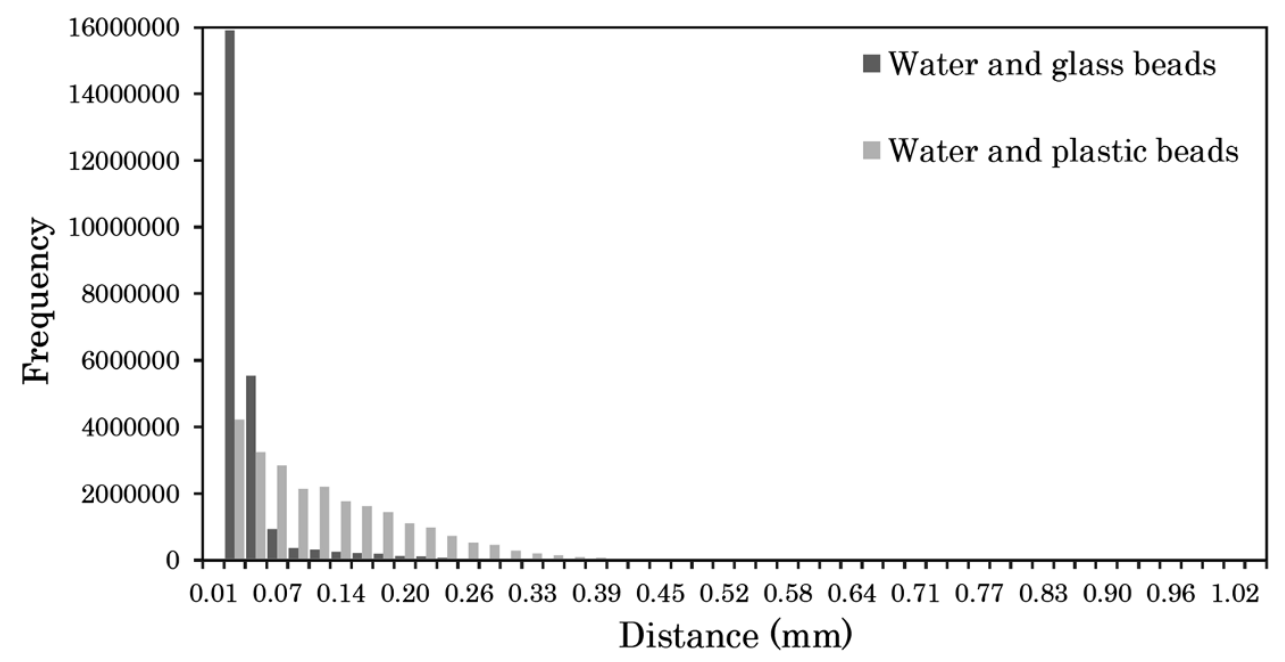


The blob analyses presented indicate that the number of trapped water blobs at the end of kerosene injection was 2.4 times greater than that of the oil blobs at the end of water injection, for similar residual saturations. Moreover, the single largest oil blob found at residual oil saturation was larger than any trapped water blob, suggesting that water remained trapped at the crevices of grain-to-grain contacts and therefore a more dominant wetting phase in this mixed-wet system. In addition, most of the blobs fell in a size range between 0.0001 and $0.001 \mathrm{~mm}^{3}$, making them 100 times smaller than those in the single wettability case.

The cumulative volume plot shows that oil blobs in range of $0.01-1 \mathrm{~mm}^{3}$ and water blobs in range of $0.001-0.1 \mathrm{~mm}^{3}$ account for an important portion of the total residual fluid trapped in the pore space. This result is consistent to the finding of Salathiel et al. (1973), whereby the smaller pores are occupied by water, whereas the oil preferentially wets the interconnected larger pores. The trend of surface area to blob volume for both oil and water was very similar, with a slight tendency of water blobs to reach a higher surface area for the same blob volume which can be attributed to stronger overall water wetting affinity to the solid surface than that of oil.

Moreover, the explicit imaging of plastic and glass beads was used to evaluate the wetting affinity of fluid to preferential solid surface by computing the shortest distance from each fluid surface (oil or water blobs) to a particular solid type (plastic or glass beads). The results confirm that oil blobs tend to adhere to plastic surfaces rather than to glass whereas water blobs tend to adhere to glass rather than to plastic. Results also suggest that glass beads have a stronger wetting affinity to water than that of plastic to oil, which is indicated by a narrower characteristic distance between water and glass surfaces.

The average percent saturation showed that irreducible water saturation tends to decrease with increasing oil wettability. In the case of the residual oil saturation, the mixed-wet sample had a trapped oil average of $16.39 \%$, whereas the residual oil in the water-wet system and the oil-wet system was found to be 17.72 and $23.86 \%$, respectively. Results from this investigation confirm that global phase trapping in porous media (residual saturation) is sensitive to the wetting characteristics of the solid matrix, but less intuitive is the evidence that the spatial distribution, characteristic size, and surface area of trapped fluid structures can vary drastically as a result of local wetting properties. These differences are then manifested in the magnitude and hysteretic attributes of capillary pressure curves that are so critical for the description of transport properties of natural permeable media.
Open Access This article is distributed under the terms of the Creative Commons Attribution License which permits any use, distribution, and reproduction in any medium, provided the original author(s) and the source are credited.

\section{References}

Al-Futaisi A, Patzek TW (2003) Impact of wettability alteration on two-phase flow characteristics of sandstones: a quasi-static description. Water Resour Res 39(2):1042. doi:10.1029/2002 wr001366

Al-Raoush RI, Willson CS (2005) A pore-scale investigation of a multiphase porous media system. J Contam Hydrol 77(1-2): 67-89. doi:10.1016/j.jconhyd.2004.12.001

Anderson WG (1987) Wettability literature survey-part 6: the effects of wettability on waterflooding. J Petrol Technol 39:1605-1622. doi:10.2118/16471-PA

Chaouki J, Larachi F, Duduković MP (1997) Noninvasive tomographic and velocimetric monitoring of multiphase flows. Ind Eng Chem Res 36(11):4476-4503. doi:10.1021/ie970210t

Culligan KA, Wildenschild D, Christensen BSB, Gray WG, Rivers ML (2006) Pore-scale characteristics of multiphase flow in porous media: a comparison of air-water and oil-water experiments. Adv Water Resour 29(2):227-238. doi:10.1016/j. advwatres.2005.03.021

Dandekar AY (2006) Petroleum reservoir rock and fluid properties. FL: CRC/Taylor \& Francis, Boca Raton

Delshad M, Najafabadi NF, Anderson GA, Pope GA, Sepehrnoori K (2006) Modeling wettability alteration in naturally fractured reservoirs. SPE/DOE Symp Improv Oil Recovery. doi:10.2118/ 100081-MS

Denison C, Carlson WD, Ketcham RA (1997) Three-dimensional quantitative textural analysis of metamorphic rocks using highresolution computed X-ray tomography: part I. Methods and techniques. J Metamorph Geol 15(1):29-44. doi:10.1111/j.15251314.1997.00006.x

Donaldson EC, Thomas RD, Lorenz PB (1969) Wettability determination and its effect on recovery efficiency. Soc Petrol Eng $\mathbf{J}$ 9:13-20. doi:10.2118/23

Heiba AA, Davis HT, Scriven LE (1983) Effect of wettability on twophase relative permeabilities and capillary pressures. In: SPE Annual Technical Conference and Exhibition, 5-8 October 1983. Society of Petroleum Engineers, San Francisco, California. doi: $10.2118 / 12172-M S$

Jackson MD, Valvatne PH, Blunt MJ (2003) Prediction of wettability variation and its impact on flow using pore-to reservoir-scale simulations. J Petrol Sci Eng 39(34):231-246. doi:10.1016/ S0920-4105(03)00065-2

Jadhunandan PP, Morrow NR (1995) Effect of wettability on waterflood recovery for crude-oil brine rock systems. SPE Reserv Eng 10:40-46. doi:10.2118/22597-PA

Jiang Z, Wu K, Couples G, van Dijke MIJ, Sorbie KS, Ma J (2007) Efficient extraction of networks from three-dimensional porous media. Water Resour Res 43(12):W12S03. doi:10.1029/ 2006wr005780

Karpyn ZT, Piri M, Singh G (2010) Experimental investigation of trapped oil clusters in a water-wet bead pack using X-ray microtomography. Water Resour Res 46(4):W04510. doi:10. 1029/2008wr007539

Ketcham RA, Carlson WD (2001) Acquisition, optimization and interpretation of X-ray computed tomographic imagery: applications to the geosciences. Comput Geosci 27(4):381-400. doi:10.1016/s0098-3004(00)00116-3 
Kumar M, Senden TJ, Latham S, Sheppard AP, Knackstedt MA, SPE Australia National University, Cinar Y, SPE, Pinczewski WV (2008) Designing for mixed wettability. Paper presented at the SPE/DOE symposium on improved oil recovery

Landry CJ, Karpyn ZT, Piri M (2011) Pore-scale analysis of trapped immiscible fluid structures and fluid interfacial areas in oil-wet and water-wet bead packs. Geofluids 11(2):209-227. doi:10. 1111/j.1468-8123.2011.00333.x

Lebedeva EV, Fogden A (2011) Micro-CT and wettability analysis of oil recovery from sand packs and the effect of waterflood salinity and kaolinite. Energy Fuels 25(12):5683-5694. doi:10.1021/ ef201242s

Lenormand R, Zarcone C, Sarr A (1983) Mechanisms of the displacement of one fluid by another in a network of capillary ducts. J Fluid Mech 135:337-353

Masalmeh SK (2003) The effect of wettability heterogeneity on capillary pressure and relative permeability. J Petrol Sci Eng 39:399-408. doi:10.1016/S0920-4105(03)00078-0

McCaffery FG, Bennion DW (1974) Effect of wettability on 2-phase relative permeabilities. J Can Petrol Technol 13:42-53. doi:10. 2118/74-04-04

Morrow NR, Mason G (2001) Recovery of oil by spontaneous imbibition. Curr Opin Colloid Interface Sci 6(4):321-337. doi:10/1016/S1359-0294(01)00100-5

Oren PE, Bakke S, Arntzen OJ (1997) Extending predictive capabilities to network models. Paper presented at the SPE annual technical conference and exhibition, San Antonio, 5-8 Oct 1997

Otsu N (1979) A threshold selection method from gray-level histograms. IEEE Trans Syst Man Cybern 9(1):62-66 (citeulike-article-id:1116982)

Patzek TW (2001) Verification of a complete pore network simulator of drainage and imbibition. Soc Petrol Eng 6(2):144-156. doi:10. 2118/71310-PA

Porter ML, Wildenschild D (2010) Image analysis algorithms for estimating porous media multiphase flow variables from computed microtomography data: a validation. Study Comput Geosci 14(1):15-30. doi:10.1007/s10596-009-9130-5

Prodanović M, Lindquist WB, Seright RS (2007) 3D image-based characterization of fluid displacement in a Berea core. Adv Water Resour 30(2):214-226. doi:10.1016/j.advwatres.2005. 05.015
Radke CJ, Kovscek AR, Wong H (1992) A pore-level scenario for the development of mixed wettability in oil reservoirs. Paper presented at the SPE annual technical conference and exhibition, Washington, 4-7 Oct 1992

Raeesi B, Piri M (2009) The effects of wettability and trapping on relationships between interfacial area, capillary pressure and saturation in porous media: a pore-scale network modeling approach. J Hydrol 376(3-4):337-352. doi:10.1016/j.jhydrol. 2009.07.060

Sahimi M (1995) Flow and transport in porous media and fractured rock: from classical methods to modern approaches. WileyVCH, Weinheim, Germany

Salathiel RA (1973) Oil recovery by surface film drainage in mixedwettability rocks. J Petrol Technol 25:1216-1224. doi:10.2118/ 4104-PA

Sezgin M, Sankur B (2004) Survey over image thresholding techniques and quantitative performance evaluation. J Electron Imaging 13(1):146-165

Speight JG (2009) Enhanced recovery methods for heavy oil and tar sands. Gulf Publishing Company, USA

Suicmez VS, Piri M, Blunt MJ (2008) Effects of wettability and porelevel displacement on hydrocarbon trapping. Adv Water Resour 31(3):503-512. doi:10.1016/j.advwatres.2007.11.003

Tartakovsky AM, Meakin P (2006) Pore scale modeling of immiscible and miscible fluid flows using smoothed particle hydrodynamics. Adv Water Resour 29(10):1464-1478. doi:10.1016/j. advwatres.2005.11.014

Tiab D, Donaldson EC (2004) Petrophysics: theory and practice of measuring reservoir rock and fluid transport properties. Gulf Professional Publishing, Boston, MA

van Dijke MIJ, Piri M (2007) Introduction to special section on modeling of pore-scale processes. Water Resour Res 43(12): W12S01. doi:10.1029/2007wr006332

Wardlaw NC (1982) The effects of geometry, wettability, viscosity and interfacial tension on trapping in single pore-throat pairs. J Can Petrol Technol 21:21-27. doi:10.2118/82-03-01

Wilkinson D, Willemsen JF (1983) Invasion percolation: a new form of percolation theory. J Phys A: Math Gen 16:3365-3376. doi:10.1088/0305-4470/16/14/028

Zhao X, Blunt MJ, Yao J (2010) Pore-scale modeling: effects of wettability on waterflood oil recovery. J Petrol Sci Eng 71(3-4): 169-178. doi:10.1016/j.petrol.2010.01.011 